\section{Long-Term Myocardial Damage in Peripartum Cardiomyopathy Associated With Myocarditis}

Hiroaki Kawano, MD; Tomayoshi Hayashi, MD; Yuji Koide, MD; Koji Maemura, MD

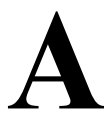
54-year-old-Japanese woman had developed peripartum cardiomyopathy (PPCM) after her third delivery at the age of 43 years. Ultrasound echocardiography (UCG) showed diffuse hypokinesis of left ventricle (LV) with an ejection fraction (LVEF) of $19 \%$. Endomyocardial biopsy showed mild cell infiltration and fibrosis (Figure A-C). On immunohistochemical staining there were more T cells (mainly CD8-positive T cells) than B cells (Supplementary Figure). Thus, she was diagnosed with PPCM related to lymphocytic myocarditis. Cardiac magnetic resonance imaging (MRI) demonstrated late gadolinium enhancement (LGE) in the LV (Figure D). One year after treatment with $\beta$-blocker, angiotensin II receptor blocker, and spironolactone, UCG indicated an LVEF of $56 \%$, and MRI LGE was decreased (Figure E). Since then, her condition had been stable under treatment with no further pregnancies. Eleven years after the onset of PPCM, cardiac MRI showed a similar level of LGE to that in the second MRI (Figure F), and LVEF was $55 \%$ on UCG. Although MRI LGE is uncommon in PPCM, ${ }^{1}$ positive
LGE and amelioration but continuation of regional LGE (myocardial damage) may be one of the characteristics of PPCM associated with myocarditis.

\section{Disclosures}

The authors declare no conflicts of interest.

\section{Reference}

1. Schelbert EB, Elkayam U, Cooper LT, Givertz MM, Alexis JD, Briller J, et al. Myocardial damage detected by late gadolinium enhancement cardiac magnetic resonance is uncommon in peripartum cardiomyopathy. J Am Heart Assoc 2017; 6: e00547.

\section{Supplementary Files}

Please find supplementary file(s):

http://dx.doi.org/10.1253/circj.CJ-19-0493

Received June 5, 2019; revised manuscript received July 3, 2019; accepted July 25, 2019; J-STAGE Advance Publication released online August 31, 2019 Time for primary review: 27 days

Department of Cardiovascular Medicine, Nagasaki University Graduate School of Biomedical Sciences, Nagasaki (H.K., Y.K., K.M.); Department of Pathology, Shimabara Prefectural Hospital, Nagasaki (T.H.), Japan

Mailing address: Hiroaki Kawano, MD, Department of Cardiovascular Medicine, Nagasaki University Graduate School of Biomedical Sciences, 1-7-1 Sakamoto, Nagasaki 852-8501, Japan. E-mail: hkawano@nagasaki-u.ac.jp

ISSN-1346-9843 All rights are reserved to the Japanese Circulation Society. For permissions, please e-mail: cj@j-circ.or.jp
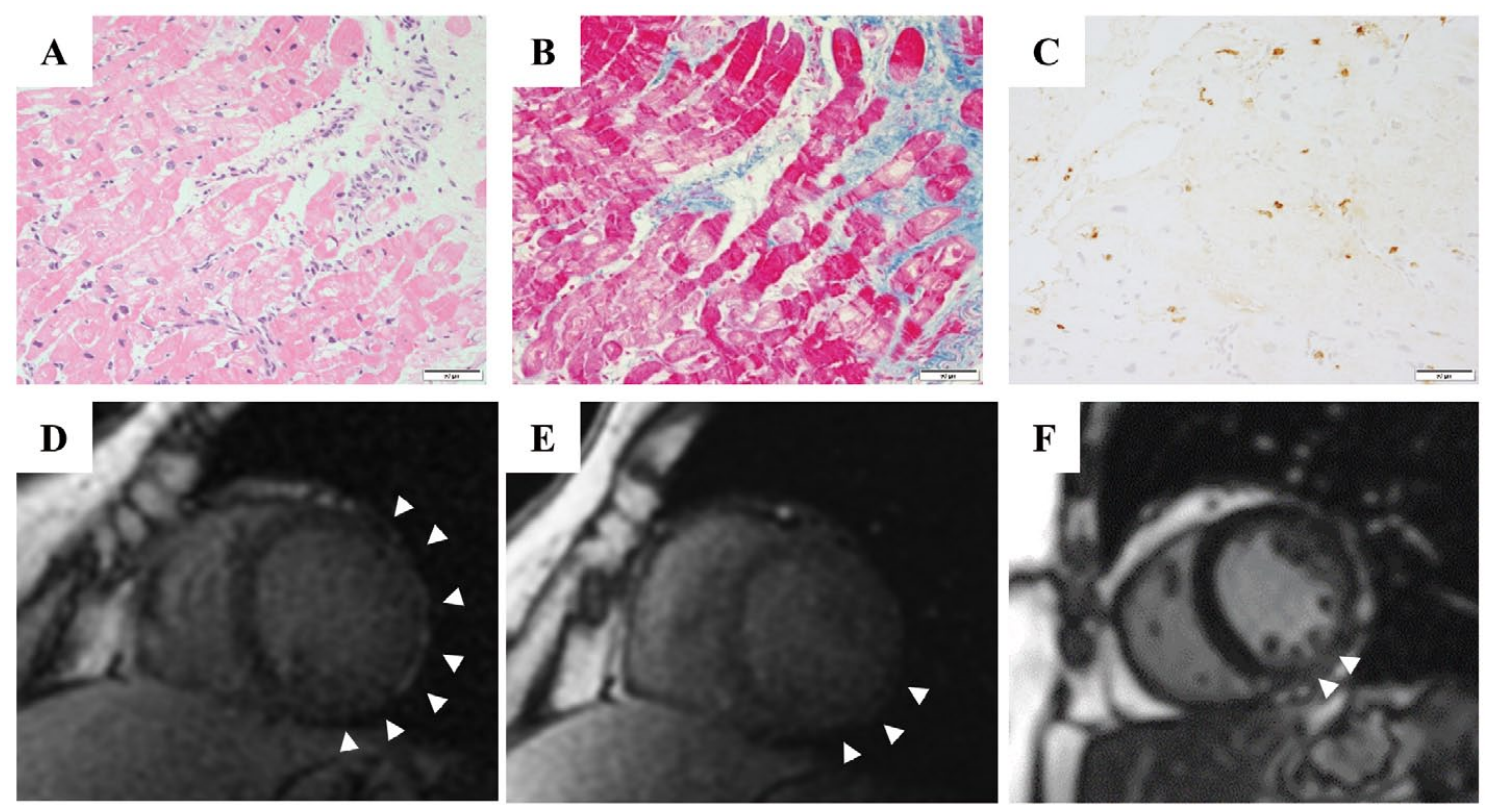

Figure. Endomyocardial biopsy showed (A) fibrosis (azan staining) and (B,C) mild cell infiltration (B, hematoxylin-eosin; C leukocyte common antigen immunostaining). (D-F) Time course on cardiac magnetic resonance imaging: late gadolinium enhancement (arrows) in the left ventricle (D) on admission; (E) 1 year later; and (F) 11 years later. 